\title{
Genetic Architecture and Dynamics of PfKelch13's Propeller Domain in Plasmodium Falciparum Clinical Isolates Collected in Senegal.
}

\section{Mariama Pouye}

Department of Animal Biology, Faculty of Science and Technology, Cheikh Anta Diop University, PO Box 5005, Dakar, Senegal.

\section{Gora Diop}

Immuno-Genetic Unit, Institut Pasteur Dakar, PO Box 220, Dakar, Senegal.

\section{celine Derbois}

Centre National de Recherche en Génomique Humaine (CNRGH), Institut de Biologie François Jacob, CEA, Université Paris-Saclay, F-91057, Evry, France.

\section{Babacar Mbengue}

Immuno-Genetic Unit, Institut Pasteur Dakar, PO Box 220, Dakar, Senegal.

\section{oumar Ka}

Immunology Service, Faculty of Medecine, Pharmacy and Dentistry-FMPO, Cheikh Anta Diop University, PO Box 5005, Dakar, Senegal.

\section{Fatou Thiam}

Department of Chemical Engineering and Applied Biology, Ecole Supérieure Polytechnique, Cheikh Anta Diop University of Dakar, PO Box 5005, Dakar, Senegal.

\section{Alassane Thiam}

Immuno-Genetic Unit, Institut Pasteur Dakar, PO Box 220, Dakar, Senegal.

\section{Laty Thiam}

G4-Malaria Experimental Genetic Approaches \& Vaccines, Pôle Immunophysiopathologie et Maladies Infectieuses, Institut Pasteur Dakar, PO Box 220, Dakar, Senegal.

\section{Eric Cabannes}

Centre National de Recherche en Génomique Humaine (CNRGH), Institut de Biologie François Jacob, CEA, Université Paris-Saclay, F-91057, Evry, France.

\section{Robert Olaso}

Centre National de Recherche en Génomique Humaine (CNRGH), Institut de Biologie François Jacob, CEA, Université Paris-Saclay, F-91057, Evry, France.

\section{Didier Menard}

Malaria Genetics and Resistance Unit, INSERM U1201, Institut Pasteur, Paris, France. 
Centre National de Recherche en Génomique Humaine (CNRGH), Institut de Biologie François Jacob, CEA, Université Paris-Saclay, F-91057, Evry, France.

Alassane Mbengue ( $\sim$ Alassane.mbengue@pasteur.sn )

G4-Malaria Experimental Genetic Approaches \& Vaccines, Pôle Immunophysiopathologie et Maladies Infectieuses, Institut Pasteur Dakar, PO Box 220, Dakar, Senegal.

\section{Alioune Dieye}

Immunology Service, Faculty of Medecine, Pharmacy and Dentistry-FMPO, Cheikh Anta Diop University, PO Box 5005, Dakar, Senegal.

\section{Research Article}

Keywords: Plasmodium, ACT, PfKelch13, clinical

Posted Date: January 20th, 2021

DOl: https://doi.org/10.21203/rs.3.rs-141363/v1

License: (c) (1) This work is licensed under a Creative Commons Attribution 4.0 International License. Read Full License 


\section{Genetic architecture and dynamics of PfKelch13's propeller domain in}

2 Plasmodium falciparum clinical isolates collected in Senegal.

3 Mariama Nicole Pouye ${ }^{1,2}$, Gora Diop ${ }^{1,3}$, Céline Derbois ${ }^{4}$, Babacar Mbengue ${ }^{3,5}$, Oumar Ka ${ }^{5}$,

4 Fatou Thiam ${ }^{6}$, Alassane Thiam ${ }^{3}$, Laty Gaye Thiam², Eric Cabannes ${ }^{4}$, Robert Olaso ${ }^{4}$, Didier

5 Menard $^{7}$, Jean-François Deleuze ${ }^{4}$, Alassane Mbengue ${ }^{2^{*}}$, Alioune Dieye ${ }^{3,5}$.

$6{ }^{1}$ Department of Animal Biology, Faculty of Science and Technology, Cheikh Anta Diop

7 University, PO Box 5005, Dakar, Senegal.

$8 \quad{ }^{2}$ G4-Malaria Experimental Genetic Approaches \& Vaccines, Pôle Immunophysiopathologie et

9 Maladies Infectieuses, Institut Pasteur Dakar, PO Box 220, Dakar, Senegal.

$10{ }^{3}$ ImmunoGenetic Unit, Institut Pasteur Dakar, PO Box 220, Dakar, Senegal.

$11{ }^{4}$ Centre National de Recherche en Génomique Humaine (CNRGH), Institut de Biologie 12 François Jacob, CEA, Université Paris-Saclay, F-91057, Evry, France.

${ }^{5}$ Immunology Service, Faculty of Medecine, Pharmacy and Dentistry-FMPO, Cheikh Anta

14 Diop University, PO Box 5005, Dakar, Senegal.

$15{ }^{6}$ Department of Chemical Engineering and Applied Biology, Ecole Supérieure Polytechnique, 16 Cheikh Anta Diop University of Dakar, PO Box 5005, Dakar, Senegal.

$17 \quad{ }^{7}$ Malaria Genetics and Resistance Unit, INSERM U1201, Institut Pasteur, Paris, France.

*Corresponding author: Alassane Mbengue (Alassane.MBENGUE@pasteur.sn)

\section{Abstract}

20 Plasmodium resistance to Artemisinin Combination-based Therapies (ACT) in Southeast 21 Asia is a major public health concern that is sporadically appearing in Africa. Senegal has 22 shifted from malaria control to elimination plans. Given notable progresses obtained through 23 robust strategic plans, it is still crucial to assess genetic variability of the Plasmodium 24 falciparum artemisinin resistance gene marker Kelch13 (PfKelch13) in circulating field 25 isolates. We here report an analysis of PfKelch13-propeller polymorphism in clinical isolates 26 collected nine years after ACT introduction in five Senegalese regions with different malaria 27 transmission settings. Sequencing of PfKelch13-propeller domain from 280 clinical isolates reveals that $16 \%(45 / 280)$ of the parasite population harbored variants. Dynamics of PfKelch13 variants reveals emerging, persistent but also disappearing mutations over time. 
In addition to the malaria epidemiology, our survey also shows the dynamics of PfKelch13

31 variants in different malaria transmission settings in Senegal. Despite the absence of

32 PfKelch13 associated artemisinin resistance mutations, a shift from $86 \%$ to $68 \%$ of

33 PfKelch13 ${ }^{\mathrm{WT}}$ was observed when comparing parasites collected prior vs. post ACT intensive

34 usage in Dakar a low malaria transmission area. All together, our data confirms the need to 35 closely monitor PfKelch13 polymorphism to anticipate and or prevent emergence of $P$. 36 falciparum resistance in Senegal.

\section{Introduction}

38 As in many other African countries, the state of Senegal has made tremendous efforts over 39 the last two decades to revert the malaria burden throughout robust control strategies. 40 Among these strategies, the intensive use of Rapid Diagnostic Tests (RDTs) as a point-of41 care diagnostic method, the large-scale distribution of bednets, as well as the progressive 42 implementation throughout the different malaria facies of vector control programs have fully 43 contributed to the notable progress recorded against malaria within Senegal. As a result, the 44 country has shifted from malaria control to elimination plans in Northern and central regions. 45 To the counter growing threat of chloroquine resistant parasites, the WHO recommended 46 artemisinin combination-based therapies (ACTs) have been progressively introduced as 47 frontline treatment regimens in late 2000s in malaria endemic countries ${ }^{1}$. In Senegal Artesunate-Amodiaquine (ASAQ) and Artemether-Lumefantrine ( $A L)$ are the first two drug combinations introduced in $\mathbf{2 0 0 6}$ as frontline treatment for uncomplicated malaria. In addition to these dual therapies, a third ACT drug regimen comprising the Dihydroartemisinin (DHA): the metabolite of all artemisinin derivatives and Piperaquine (PQ), has been slowly distributed to prevent emergence and/or spread of artemisinin resistant Plasmodium falciparum isolates. However artemisinin resistance (AR) has emerged in Southeast Asia $(\mathrm{SEA})^{2}$ since 2006 therefore becoming a global health concern. AR is defined as a reduced $P$. falciparum susceptibility towards Artemisinin derivatives manifesting with a clinical delay in parasite clearance upon malaria treatment ${ }^{3}$.

There is now evidence that AR is now due to emerging single point mutations in $P$. falciparum kelch 13 gene $^{4}$. Mutations, in the parasite gene resistant marker (PfKelch13, PF3D7_1343700), located in its propeller domain (C-terminal domain of the protein) were indeed shown to be clinically associated with a delay in parasite clearance in the first 3 days of treatment following artemisinin monotherapy or ACT. Next the elevated survival rate based on the in vitro ring survival assay (RSA $)^{5}$ was developed and subsequently used as a powerful tool to measure AR in vitro. In conjunction with PfKelch13's propeller mutations, 
both clinical parasite clearance delay and high RSA level are commonly used to define parasite resistance phenotypes in the field and in the laboratories respectively ${ }^{6}$.

PfKelch13 ${ }^{\mathrm{C} 580 \mathrm{Y}}$ and PfKelch13 $3^{\mathrm{F} 446 \mathrm{I}}$ are the most common variants in the propeller domain of the parasite gene marker known to be associated with residual and viable parasites upon ACT treatments in SEA ${ }^{7}$. These mutations were shown in addition to PfKelch $13^{\text {R539T }}$ to confer increased in vitro survival rate expressed by the RSA in both clinical and laboratory engineered strains, while parasites with PfKelch $13^{W T}$ showed a sensitivity to $D H A^{7}$. As $A R$ spreads in China Myanmar Border while a decreased in vitro artemisinin sensitivity of $P$. falciparum isolates across India was reported ${ }^{8}$. Next, isolated cases of treatment failure have been reported in other regions of the world in conjunction or independently from PfKelch $13^{9}$. All together, these findings make tracking of AR not only an urgent public health problem that needs a robust tracking system, but also a concern that does require scientific tools to provide insights on the biological relevance of each mutation. In parallel, both impact of different parasite background and the identification of other markers that could play a critical role in the artemisinin resistance mechanism will be critical to delay spread of $A R$ in malaria endemic regions.

In sub-Saharan Africa, drug clinical efficacy studies reveals that ACTs are still efficacious against $P$. falciparum parasites. Indeed, PfKelch13 genetic diversity of thousands African $P$. falciparum isolates has shown a very low frequency of SEA mutations in circulating parasites $^{10-12}$. Nevertheless, sporadic cases of either clinical treatment failure upon treatment or increased in vitro RSA levels have been documented in African background parasites ${ }^{13-15}$. The emergence of indigenous mutations, associated with ACT treatment failure cases in Equatorial Guinea in $2017^{13}$. The same year four Ugandan-imported artemether-lumefantrine treatment failure cases were reported to be independent to PfKelch13 mutations in the United Kingdom ${ }^{14}$. Next, PfKelch13 ${ }^{\text {R622I }}$ was identified in an Ethiopian patient with a persistent parasitemia at day 3 upon ACT treatment. However, it is important to note that all patients included in the study showed a parasite clearance at day 28 post treatment indicating a lack of correlation between PfKelch $13^{\mathrm{R} 6221}$ mutation and resulting phenotype ${ }^{16}$. More recently a clear early warning signs of $A R$ in Rwanda was reported with a Pfkelch $13^{\mathrm{R} 561 \mathrm{H}}$ mutation identified in 19 of $257(7.4 \%)$ included patients ${ }^{17}$

All aforementioned African studies calls for an urgent need to routinely map out the genetic diversity of the AR gene marker and to establish efficient surveillance system to closely monitor parasite clearance half-life within Sub-Saharan Africa accounting for more than $90 \%$ of total malaria death ${ }^{18}$.

A total of 280 Senegalese $P$. falciparum clinical isolates were collected between 2014 and 2015 (8-9 years after ACT introduction) from five regions with variable malaria transmission 
100 intensity. PfKelch13 genetic diversity was assessed, using Sanger sequencing, to 101 understand the dynamics of PfKelch13 mutations in Senegal. Seventy four (74) samples

102 were also collected before ACT introduction in the Urban-Senegalese capital Dakar, a region 103 that has shifted to malaria elimination plans, and PfKelch13 mutations compared to post 104 ACT samples. Our study provides a map of PfKelch13 distribution in circulating Senegalese $105 P$. falciparum clinical isolates from low to high malaria transmission areas. In addition our 106 epidemiology data on the parasitic disease shows an increase of severe malaria cases that 107 could result from the low infection rates and loss of immunity. The prior vs. post ACT 108 Pfkelch13 mutations comparison shows a useful baseline for emerging PfKelch13 109 polymorphism in parasites under 8 and 9 years ACT drug pressure in Senegal.

\section{Results:}

111 Demographic characteristic of the study population, malaria prevalence and mortality.

112 The data of our study were generated from 280 malaria-infected patients, recruited from five 113 regions of variable malaria transmission intensity. From low to high transmission setting 114 hospitals from Louga, Saint-Louis $(N=14)$, Dakar $(N=25)$, Kolda $(N=117)$ and 115 Tambacounda $(\mathrm{N}=124)$ were included respectively. In both the pre-elimination regions 116 Saint-Louis and Louga (referred as Louga in our work) the low number of malaria infections 117 (14) confirms the lowest malaria prevalence. All 280 blood samples were collected between 1182014 and 2015 i.e. 8 to 9 years following ACT introduction in Senegal. All included patients 119 were tested and confirmed to be Pf-RDT positive on collection/visit day. Both demographic 120 and clinical characteristics of the study participants are summarized in Table 1. The median 121 sex ratio was 1.52 across the five sampling sites (Chi-square, $p=0.118$ ). The age of 122 participants ranged between 0 to 85 years with a median of 17 years (Supplementary File. 1231 a). There was a significant difference in the mean age across sites (ANOVA, $p=0.03$ ) with 124 the youngest participants from Tambacounda (mean age 8 years) and the eldest in Kolda 125 (mean age, 18 years). This finding is indicating that in the highly malaria endemic sites there 126 is in addition to the vulnerable 0 to 5 years old populations, a susceptibility to malaria 127 infections in the 10 to 18 years old group. With respect to the disease outcome at inclusion 128 level, the percentage of severe malaria cases (SM) was higher in Dakar (96\%), followed by 129 Kolda (75.2\%) and Louga (64.2\%), while this outcome was lower in Tambacounda (53.2\%)

130 (Supplementary File $1 \mathrm{~b}$ ). Overall, seven participants died of malaria $(2.5 \%)$ during the 131 study period and, Dakar, once again accounted for the highest number of deaths recorded 132 across the study sites (16\%), followed by Kolda and Tambacounda (1.7 and $0.81 \%$, 133 respectively (Table 1). These results indicate, between 2014 and 2015, an increasing 134 severity of malaria infection that is probably associated with the loss of immunity in malaria 135 and decreased infection rates in low malaria endemic areas and higher transmission rate 
136 respectively. As the results our data shows that despite notable progress that Senegalese

137 populations remain at risk for severe malaria infections.

138 Geographical distribution of PfKelch13 mutations 8 and 9 years following ACT 139 introduction

140 PfKelch13 propeller domain was successfully genotyped for all the 280-collected $P$. 141 falciparum field isolates. Fifteen variable mutations were detected, with $16 \%$ of the isolates

$142(45 / 280)$ harboring at least one mutation when compared to the reference Pf3D7-PfKelch13 143 sequence from plasmdoDB (PF3D7_1343700). Seven of these mutations were synonymous, 144 while the remaining eight were non-synonymous. Of all non-synonymous mutations reported 145 here, the N689Y mutation was the most frequent variant (48.33\%), followed by C447Y 146 (18.33\%) and V666I, while D252N, A282T, L678F and N689C were present at similar 147 proportions (1.67\%) (Fig. 1-a). PfKelch13 ${ }^{\mathrm{A} 578 \mathrm{~S}}$ the frequent African background mutation 148 known not to confer $\mathrm{AR}^{10}$ was detected at a relatively low frequency (3.33\%). The respective 149 positions of these individual mutations within PfKelch13 propeller domain are depicted in Fig.

150 1-b. None of PfKelch13 mutations previously associated with delay in parasite or increased 151 level of in vitro survival rate (RSA) was found in these 280 post ACT (2014 and 2015) 152 samples. The geographical map indicates a high prevalence of PfKelch $13^{\mathrm{WT}}$ isolates across 153 the study sites between 2014 and 2015 (Fig. 1-c). Across all sites, Louga and Dakar had the 154 least diverse parasites populations with respectively one and three PfKelch13 variants 155 detected, while Kolda and Tambacounda presented the most highly diverse parasite 156 populations with respectively seven and eleven PfKelch13 variants reported (Sup. file 157 2). Eleven samples were found to harbor multiple PfKelch13 variants, of these, five samples 158 had the combination N689Y/C447Y, two had V666I/G690G and one had N689Y/V510V, 159 respectively, while one and two samples harbored the following variant combinations, $160 \mathrm{D} 452 \mathrm{~N} / \mathrm{A} 582 \mathrm{~T} / \mathrm{N689Y/C447Y} \mathrm{and} \mathrm{G690G/N689Y/C447Y,} \mathrm{respectively.} \mathrm{Further} \mathrm{analysis}$ 161 revealed no association between PfKelch13 variants and disease severity or deaths, as only $1620.14 \%(4 / 280)$ of the enrolled patients infected with mutant PfKelch13 isolates died of malaria 163 (Sup. file 2). Among all sites the area of low transmission and low malaria immunity city and 164 capital Dakar had a higher rate of PfKelch13 polymorphism (24\%) (Fig. 1-d) although none 165 of documented PfKelch13 and Artemisinin resistance associated mutations was detectable.

166 Dynamics of PfKelch13 polymorphism in Dakar across time (Prior vs. Post ACT 167 introduction).

168 The prevalence of PfKelch13 mutations was also assessed across time using samples 169 collected from Dakar. Here we sought to understand the profile of emerging mutations in the 170 parasites by comparing clinical isolates collected between 2004 and 2005 i.e two and one 
171 year prior to the introduction of ACTs as frontline treatment regimens in Senegal and the

172 previously analyzed samples from Dakar (collected 8 to 9 years post ACT introductions). The

173 pre-ACT samples were made of 74 samples obtained from patients with a median age of 27

174 years (range 2-74 years) (Table 2). Of these samples, 36 were collected from patients with

$175 \mathrm{UM}$, while the remaining 38 patients suffered from SM. Our analysis revealed a significantly

176 lower prevalence of SM cases in the pre-ACT $(51.3 \%)$ samples as compared to the post-

177 ACT samples (96\%) (Supplementary file. 2). Further analysis of the patients' clinical issue

178 revealed that $20 \%$ (15/74) died from the malaria onset (Table 1$)$. This further emphasizes the

179 sharp decrease in malaria-associated mortality in the 2014-2015 cohort where only $2.5 \%$

180 mortality was recorded (Tables $1 \& 2$ ).

181 PfKelch13 genotyping revealed five mutations from the pre-ACT samples. These comprised

182 two synonymous (A504A and G638G) and three non-synonymous mutations (A578S, G639C

183 and N689Y). Of these, only two (A578S and N689Y) have persisted in the parasites

184 population over the covered period of study. Indeed both variants were found in our post-

185 ACT samples, while the remaining three mutations (A504A; G638G and G639C)

186 disappeared along with ACT drug pressure (Table 3). Interestingly our retrospective reveals

187 emergence of the C447Y under ACTs drug pressure (0 to $8 \%$ ). This variant replacement of a

188 cysteine to a tyrosine is similar to the most frequent C580Y mutation known to be associated

189 with delay in parasite clearance and in vitro high survival rate. Moreover, it is important to

190 mention that the prevalence of the $\mathrm{K} 13^{\mathrm{WT}}$ variant shifted from 86 to $68 \%$ in Dakar (Fig. 1-c \&

191 Fig. 2).

192 Discussion:

193 The growing threat associated to AR calls for an urgent need to monitor its potential spread

194 or emergence in Africa, where over $90 \%$ of the global malaria cases occur 195 yearly ${ }^{18}$. Resistance to artemisinin has first been reported from SEA ${ }^{2}$ where resistance to 196 most antimalarials has been always firstly described before its spread to other malaria 197 endemic regions. Although AR associated mutations are yet to be declared in Africa, there 198 are increasing studies describing appearance of either SEA associated mutations in African 199 settings or emerging mutations causing worrying delay in parasite clearance upon ACT 200 treatments $^{13-15,17}$. In Africa tremendous efforts have been done to reduce malaria burden. We 201 are currently facing new challenges that if not controlled might postpone the malaria 202 elimination plans adopted by few countries including Senegal. The possibility that AR could emerge independently in Africa makes its surveillance crucial. Our study provides a geographical mapping of $\mathrm{K} 13$ variants in four regions with variable malaria transmission

205 settings. With fifteen mutations in PfKelch13-propeller domain of 280 clinical isolates 206 collected 8 to 9 after ACT introduction we are adding valuable information for the antimalarial 
tracking strategies of the Senegalese National malaria control program. None of the SEA AR 208 or newly PfKelch13 variant found in Africa and reported to be causing delayed parasite 209 clearance upon ACT treatment was detected in our samples. This is in phase with previous 210 reports indicating the absence of SEA-associated mutations in Senegal ${ }^{19-24}$. Both the malaria 211 epidemiology and physiopathology of the tropical disease reveal an increasing proportion of 212 severe malaria that results from a loss of malaria immunity in low malaria transmission 213 settings. Indeed, the highest rate of PfKelch13 polymorphism was found in Dakar which is an 214 area of low transmission and low malaria immunity city ${ }^{25}$. Although none of documented 215 PfKelch13 and AR associated mutations was detectable, this finding is in phase with 216 previous observations corroborating historical observations of antimalarial resistance in low 217 malaria transmission areas $^{26}$. The frequency of severe malaria cases in the 2014-2015 218 cohort is confirming a loss of malaria immunity as seen in Dakar and Louga. It is important to 219 note that we found emerging PfKelch13 in other regions with high incidence. Overall these 220 results illustrate the plasticity of the malaria genome of parasite circulating in Senegal. This 221 phenomenon is confirmed in the parasites collected priory to ACT introduction in Senegal. 222 Comparison of PfKelch13 mutations pre vs. post ACT indicates the impact of ACT drug 223 pressure on PfKelch13 genetic diversity. Contrarily to PfKelch13 ${ }^{\text {A578S }}{ }^{\text {N689Y }}$ and C447Y the 224 variants PfKelch13 ${ }^{\text {A504A, G638G }}$ and ${ }^{\text {G639C }}$ did not persist over time. Among all these mutations 225 PfKelch $13^{\mathrm{C} 447 \mathrm{Y}}$ is the only variant that has clearly emerged under ACT drug pressure. Overall 226 our study brings insights on the dynamics of PfKelch13 mutations in parasite circulating in 227 Senegal. So far no clinical delay in parasite clearance was reported in Senegal ${ }^{23}$. This is 228 confirming that ACTs remain efficacious in Senegal. The fact that none of the SEA229 artemisinin resistance-associated mutations were found among clinical isolates under 10 230 years of drug pressure collected samples indicates that despite the isolated cases of AR in 231 Africa and the usage of the three ACT drug regimen the Senegalese background parasites 232 are still sensitive to the cornerstone of antimalarial drugs. However given both the low 233 malaria transmission settings and the loss of immunity known to give rise to a more rapid 234 expansion of artemisinin-resistant parasites we need to strengthen the surveillance system to 235 anticipate antimalarial resistance in Senegal against the cornerstone of all antimalarial 236 currently available on the market.

\section{Methods:}

\section{Sample collection and processing}

239 The study was done in accordance to the guidelines of the Institutional Review Board (IRB) 240 of University Cheikh Anta Diop Dakar Senegal and on behalf of the National Research 241 Ethics Committee Clear statement of approval for human sampling was obtained for the 
2004-2005 and 2014-2015 studies. The identification protocol Numbers are Protocol

243 0089/2005/CER and Protocol No 001/2015/CER/UCAD respectively. Informed written and

244 signed consent was obtained from all participants or their legal guardians for all participants

245 under the age of 18 years old. Local translators were recruited in each regions to make sure

246 that all participants or legal tutors were well informed and therefore aware of the study

247 contributions in the National malaria control program, Malaria positive patients were

248 recruited from areas varying transmission intensities.

249 Kolda and Tambacounda present the highest transmission intensity, while the two Northern 250 regions Saint Louis and Louga (referred to hereafter as Louga), where malaria pre251 elimination campaigns are being launched ${ }^{27}$, were included as lowest malaria transmission 252 intensity areas. The transmission intensity in Dakar-Diamniadio lies somewhere between 253 these two extremes. Sampling was undertaken in regional hospitals of each region. In Dakar 254 the study was done in collaboration with the Principal Military Hospital (HPD) and 255 Diamniadio Children Hospital (DCH). Samples from these two centers will subsequently be 256 referred to as Dakar as they are both located within the same region the urban Senegalese 257 capital. Venous blood samples were collected in EDTA tubes for each enrolled patient at 258 admission day before treatment. Malaria diagnosis was performed by a rapid diagnostic test 259 (SD BIOLINE Malaria Ag P.f) provided to all health services by the National Malaria Control 260 program. All P. falciparum positive samples were further confirmed by microscopy. Severity 261 of infection was defined by clinicians following WHO guidelines and eligibility criteria ${ }^{28}$. 262 Uncomplicated malaria (UM) and severe malaria cases (SM) were reported for each malaria 263 infected patient.

\section{DNA extraction and PfKelch13 genotyping}

265 Plasmodium genomic DNA was extracted from blood of all confirmed malaria infected 266 patients, using Qiagen Kit following manufacturer's instructions. Nested PfKelch13 PCR was 267 carried out to amplify the propeller domain of the gene. First amplification was done using the 268 primary primer set PfKelch13_PCR_F 5'-CGGAGTGACCAAATCTGGGA-3' and 269 PfKelch13_PCR_R 5'-GGGAATCTGGTGGTAACAGC-3'. The resulting products were used 270 as matrices for the PfKelch13 nested amplification. The nested amplification was carried out 271 for the propeller domain with PfKelch13_N1_F 5'-GCCAAGCTGCCATTCATTTG-3' and 272 PfKelch13_N1_R 5'-GCCTTGTTGAAAGAAGCAGA-3' primers ${ }^{4}$. PCR reactions were 273 prepared in a final volume of $25 \mu$ l comprising $13.75 \mu$ l of nuclease-free water, $0.625 \mu$ l of each 274 primer, $5 \mu \mathrm{l}$ of $5 \mathrm{x}$ HOT FirePol Master Mix and $5 \mu \mathrm{l}$ of gDNA for each reaction. Thermocycling 275 conditions were carried out with an initial denaturation at $95^{\circ} \mathrm{C}$ for 15 minutes, followed by 27630 cycles with 30 seconds denaturation at $95^{\circ} \mathrm{C}, 2$ minutes annealing at $58^{\circ} \mathrm{C}, 2$ minutes 
extension at $72^{\circ} \mathrm{C}$; and a final 10 minutes extension at $72^{\circ} \mathrm{C}$ for the first PCR. The nested

278 PCR was performed using the following settings, 15 minutes initial denaturation at $95^{\circ} \mathrm{C}$

279 followed by 40 cycles with 30 seconds denaturation at $95^{\circ} \mathrm{C}, 1$ minute of 40 cycles annealing

280 at $60^{\circ} \mathrm{C}, 1$ minute of 40 cycles extension at $72^{\circ} \mathrm{C}$ and a final 10 minute extension at $72^{\circ} \mathrm{C}$.

281 PCR products were sequenced (Sanger) and analysed with the Genalys software version

$2822.0 b^{29}$.Sequencing reactions were performed according to the Dye terminator v3.1 method 283 using an ABI PRISMs 3730 DNA Analyzer (Applied Biosystems, Foster City, CA, USA). Mix 284 sequencing contained $2 \mu \mathrm{l} \mathrm{PCR}$ product, $0.5 \mu$ l BigDye $v 3.1,1.87 \mu$ l buffer $5 \mathrm{X}, 0.5 \mu \mathrm{l}$ primers $285(10 \mu \mathrm{m})$, up to $5.17 \mu \mathrm{l}$ with water. Conditions were set as follows, $96{ }^{\circ} \mathrm{C}$ for $5 \mathrm{mn}$, 25 cycles of $28696^{\circ} \mathrm{C}, 10 \mathrm{sec} ; 60^{\circ} \mathrm{C}$ for $4 \mathrm{mn}$ and $15^{\circ} \mathrm{C}$ forever. Sequencing products were purified with 287 Sephadex G50 superfine (GE Healthcare). The resulting sequences were next analysed and 288 the corresponding chromatograms closely checked for mutation detections. All nucleotide 289 blasts were done using NCBI blast and Plasmodb 3D7 (PlasmoDB 46 version released Nov 290 6th 2019) (www.plasmodb.org). One-way ANOVA was performed using GraphPad Prism 291 version 8.0.0 (GraphPad Software, San Diego, California USA, www.graphpad.com).

\section{References}

1. World Health Organization. Global Partnership to Roll Back Malaria. Antimalarial drug 294 combination therapy : report of a WHO technical consultation, 4-5 April 2001. (2001).

2. Noedl, H. et al. Evidence of artemisinin-resistant malaria in western Cambodia. N. Engl. J. Med. 359, 2619-2620 (2008).

3. Dondorp, A. M. et al. Artemisinin resistance in Plasmodium falciparum malaria. N. Engl. J. Med. 361, 455-467 (2009).

4. Ariey, F. et al. A molecular marker of artemisinin-resistant Plasmodium falciparum malaria. Nature 505, 50 (2014).

5. Witkowski, B. et al. Novel phenotypic assays for the detection of artemisinin-resistant Plasmodium falciparum malaria in Cambodia: in-vitro and ex-vivo drug-response studies. Lancet Infect. Dis. 13, 1043-1049 (2013).

304 6. Fairhurst, R. M. \& Dondorp, A. M. Artemisinin-resistant Plasmodium falciparum malaria. $305 \quad$ Emerg. Infect. 10 409-429 (2016). 
7. Imwong, M. et al. Molecular epidemiology of resistance to antimalarial drugs in the Greater Mekong subregion: an observational study. Lancet Infect. Dis. 20, 1470-1480 (2020).

8. Zhang, J. et al. In vitro susceptibility of Plasmodium falciparum isolates from the ChinaMyanmar border area to artemisinins and correlation with K13 mutations. Int. J. Parasitol. Drugs Drug Resist. 10, 20-27 (2019).

9. Mukherjee, A. et al. Artemisinin resistance without pfkelch13 mutations in Plasmodium falciparum isolates from Cambodia. Malar. J. 16, 1-12 (2017).

10. Kamau, E. et al. K13-propeller polymorphisms in Plasmodium falciparum parasites from sub-Saharan Africa. J. Infect. Dis. 211, 1352-1355 (2014).

11. Ménard, D. et al. A Worldwide Map of Plasmodium falciparum K13-Propeller Polymorphisms. N. Engl. J. Med. 374, 2453-2464 (2016).

12. Haldar, K., Bhattacharjee, S. \& Safeukui, I. Drug resistance in Plasmodium. Nat. Rev. Microbiol. 16, 156 (2018).

13. Lu, F. et al. Emergence of indigenous artemisinin-resistant Plasmodium falciparum in Africa. N. Engl. J. Med. 376, 991-993 (2017).

14. Sutherland, C. J. et al. pfk13-independent treatment failure in four imported cases of Plasmodium falciparum malaria treated with artemether-lumefantrine in the United Kingdom. Antimicrob. Agents Chemother. 61, (2017).

15. Ikeda, M. et al. Artemisinin-resistant Plasmodium falciparum with high survival rates, Uganda, 2014-2016. Emerg. Infect. Dis. 24, 718 (2018).

16. Bayih, A. G. et al. A Unique Plasmodium falciparum K13 Gene Mutation in Northwest Ethiopia. Am. J. Trop. Med. Hyg. 94, 132-135 (2016).

17. Uwimana, A. et al. Emergence and clonal expansion of in vitro artemisinin-resistant Plasmodium falciparum kelch13 R561H mutant parasites in Rwanda. Nat. Med. 26, $1602-1608$ (2020).

18. WORLD HEALTH ORGANIZATION. WORLD MALARIA REPORT 2019. (WORLD HEALTH ORGANIZATION, 2019). 
19. Torrentino-madamet, M. et al. Limited polymorphisms in k13 gene in Plasmodium falciparum isolates from Dakar, Senegal in $2012-2013.1-5$ (2014).

20. Boussaroque, A. et al. Emergence of Mutations in the K13 Propeller Gene of Plasmodium falciparum Isolates from Dakar, Senegal, in 2013-2014. Antimicrob. Agents Chemother. 60, 624-627 (2016).

21. Madamet, M. et al. Absence of association between polymorphisms in the K13 gene and the presence of Plasmodium falciparum parasites at day 3 after treatment with artemisinin derivatives in Senegal. Int. J. Antimicrob. Agents 49, 754-756 (2017).

22. Talundzic, E. et al. Molecular epidemiology of Plasmodium falciparum kelch13 mutations in Senegal determined by using targeted amplicon deep sequencing. Antimicrob. Agents Chemother. 61, (2017).

23. Diallo, M. A. et al. Efficacy and safety of artemisinin-based combination therapy and the implications of Pfkelch13 and Pfcoronin molecular markers in treatment failure in

$$
\text { Senegal. Sci. Rep. 10, 1-8 (2020). }
$$

24. Gaye, A. et al. Amplicon deep sequencing of kelch13 in Plasmodium falciparum isolates from Senegal. Malar. J. 19, 1-8 (2020).

25. Henry, M. et al. Urban malaria in Dakar, Senegal: chemosusceptibility and genetic diversity of Plasmodium falciparum isolates. Am. J. Trop. Med. Hyg. 75, 146-151 (2006).

26. Scott, N. et al. Implications of population-level immunity for the emergence of artemisinin-resistant malaria: a mathematical model. Malar. J. 17, 279 (2018).

27. PNLP. Bulletin épidemiologique annuel. (2016).

28. Saissy, J. M., Rouvin, B. \& Koulmann, P. Severe malaria in intensive care units in 2003. Médecine Trop. Rev. Corps Santé Colon. 63, 258-266 (2003).

29. Takahashi, M., Matsuda, F., Margetic, N. \& Lathrop, M. Automated identification of single nucleotide polymorphisms from sequencing data. J. Bioinform. Comput. Biol. 1, 253-265 (2003).

\section{Acknowledgements}


We would like to thank the Genopole team for the sequencing; Dr Shahir RIZK for making available to us PyMol software; the study participants and field teams for their involvement in the study.

\section{Author's contribution}

365 A.D. and D.M. conceived the study design. M.N.P., G.D. and A.M. wrote the manuscript. M.N.P. C.D., E.C., R.O. and J.F.D. performed the experiments. M.N.P. and A.M. made the data analysis. B.M. and O.K. did the samples collection. F.T. and A.T. were in charge of data management. L.G.T. did the statistical analysis.

\section{Competing interests}

The authors declare no competing interests.

Table 1: Demographic characteristics of the study population between 2014 and 2015 for PfKelch13 mapping in Senegal. Clinical symptoms of all 280 included patients are indicated. Uncomplicated or mild malaria (UM) and severe malaria (SM) cases are counted for each region. Median ages, sex group, issue of malaria infection are also listed. ND corresponds patients for whom we miss their follow up information. These patients were based in Tambacounda areas. A sex ration of 1.52 was obtained across samples. * represents the 7 and 6 missing sex and age information respectively.

Figure 1: Geographical distribution of PfKelch13 mutations between 2014 and 2015. a. 15 variants found in 280 clinical isolates are represented. Synonymous mutations are written in black and nonsynonymous mutations are in red. $b$. Location of the 7 Ns-SNPs on the propeller domain are shown using PfKelch13 structure pdb code: 4ZGC. c. Mapping of PfKelch13 SNPs in our study sites reveals a high frequency of mutations in highly endemic regions (Kolda and Tambacounda). PfKelch13 ${ }^{\mathrm{WT}}$ (blue) remains highly frequent in all sites indicating efficacy of ACTs in collected parasite lines. $d$. Frequency of all SNPs per region is shown. Dakar the capital and low malaria endemic city shows a higher frequency of SNPs with $24 \%$.

Table 2: Demographic characteristics of the study population priory to ACT introduction in Dakar (between 2004 \& 2005). Clinical symptoms of all 74 included patients are indicated. Median ages, sex group, issue of malaria infection are also listed. ND corresponds to missing sex information. With $20 \%$ of patients who died from malaria population from Dakar was at higher malaria risk before ACT introductions.

Figure 2: PfKelch13 polymorphism in Dakar in 2004-2005. Prevalence of PfKelch13 priory to ACT introduction reveals in high frequency of PfKelch $13^{\mathrm{WT}}$ (85.33\%). Mutations including A578S, N689Y, A504A C638G and G639C were detectable. None if these variant is associated with artemisinin resistance. 
Table 3: Evolution of PfKelch13 mutations in $P$. falciparum isolates collected in Dakar 2004-2005 vs. 2014-2015. Dynamic of PfKelch13 SNP prior vs. post ACT introductions. The frequency of PfKelch $13^{\text {WT }}$ has shifted from 86 to $68 \%$. Among all mutations PfKelch $13^{\mathrm{C} 447 \mathrm{Y}}$ is the only variant, which has emerged under ACT drug pressure. PfKelch13 ${ }^{A 504 C}$, PfKelch13 ${ }^{\mathrm{G} 638 \mathrm{G}}$, PfKelch13 ${ }^{\mathrm{G} 639 \mathrm{C}}$ have disappeared over the years while PfKelch $13^{\mathrm{A} 580 \mathrm{~S}}$ and PfKelch $13^{\mathrm{N} 688 \mathrm{Y}}$ are still maintained in the genetic of the gene resistance marker.

Table and Figures:

Table 1: Demographic characteristics of the study population between 2014 and 2015 for PfKelch13 mapping in Senegal.

\begin{tabular}{|c|c|c|c|c|c|c|}
\hline \multirow{2}{*}{\multicolumn{2}{|c|}{\begin{tabular}{|l|} 
Number of subjects \\
\end{tabular}}} & Louga & Dakar & Tambacounda & Kolda & Total \\
\hline & & 14 & 25 & 124 & 117 & 280 \\
\hline \multicolumn{2}{|c|}{$\begin{array}{l}\text { Median Age (years old) } \\
\left(\text { min-max) }{ }^{*}\right.\end{array}$} & $\begin{array}{c}10 \\
(4-51)\end{array}$ & $\begin{array}{l}11,5 \\
(1-61)\end{array}$ & $\begin{array}{c}8 \\
(0-84)\end{array}$ & $\begin{array}{c}18 \\
(0-85)\end{array}$ & $\begin{array}{c}17 \\
(0-85)\end{array}$ \\
\hline \multirow{4}{*}{ Sex Group } & Male (M) & 10 & 17 & 55 & 60 & 142 \\
\hline & Female (F) & 4 & 8 & 62 & 57 & 131 \\
\hline & ND & 0 & 0 & 7 & 0 & 7 \\
\hline & Ratio (M/F) & 2.5 & 2.1 & $0.9^{*}$ & 1 & $1.1^{*}$ \\
\hline \multirow{2}{*}{$\begin{array}{l}\text { Clinical } \\
\text { outcomes }\end{array}$} & UM (\%) & $5(35.7)$ & $1(4)$ & $58(46.8)$ & $29(24.8)$ & $93(33.2)$ \\
\hline & SM (\%) & $9(64.3)$ & $24(96)$ & $66(53.2)$ & $88(75.2)$ & $187(66.8)$ \\
\hline \multirow{3}{*}{ Issue } & Survival (\%) & $14(100)$ & $21(84)$ & $116(93.5)$ & $115(98.3)$ & $266(95)$ \\
\hline & Died (\%) & 0 & $4(6)$ & $1(0.8)$ & $2(1,7)$ & $7(2.5)$ \\
\hline & ND (\%) & 0 & 0 & $7(5.7)$ & 0 & $7(2.5)$ \\
\hline
\end{tabular}


Figure 1: Geographical distribution of PfKelch13 mutations between 2014 and 2015.
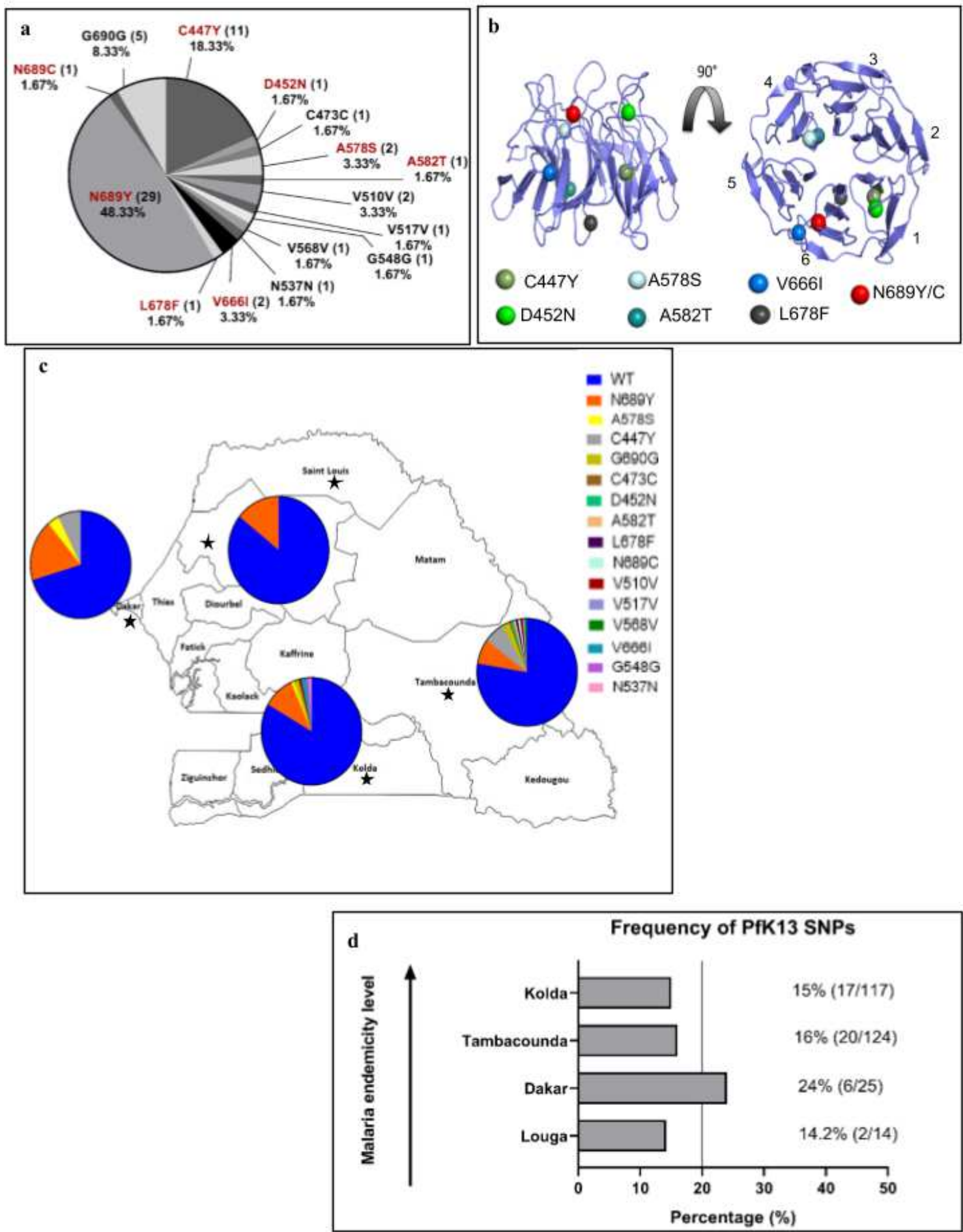
413 Table 2: Demographic characteristics of the study population priory to ACT 414 introduction in Dakar (between 2004 \& 2005).

\begin{tabular}{|cc|c|}
\hline \multicolumn{3}{|c|}{ Pre ACT Samples (Dakar) } \\
\hline \multicolumn{2}{|c|}{$\begin{array}{c}\text { Number of subjects } \\
\text { Median Age (years old) } \\
\text { (min-max) }\end{array}$} & $\begin{array}{c}27 \\
(2-74)\end{array}$ \\
\hline \multirow{2}{*}{ Sex Group } & Male (M) & 40 \\
\cline { 2 - 3 } & Female (F) & 27 \\
\cline { 2 - 3 } & ND & 7 \\
\cline { 2 - 3 } Clinical & Ratio (M/F) & 1,5 \\
\cline { 2 - 3 } outcomes & UM (\%) & $36(48.6)$ \\
\hline \multirow{2}{*}{ Issue } & SM (\%) & $38(51.4)$ \\
\cline { 2 - 3 } & Survival (\%) & $59(80)$ \\
\cline { 2 - 3 } & Died (\%) & $15(20)$ \\
\cline { 2 - 3 } & ND & 0 \\
\hline
\end{tabular}

415 Figure 2: PfKelch13 polymorphism in Dakar in 2004-2005.

416

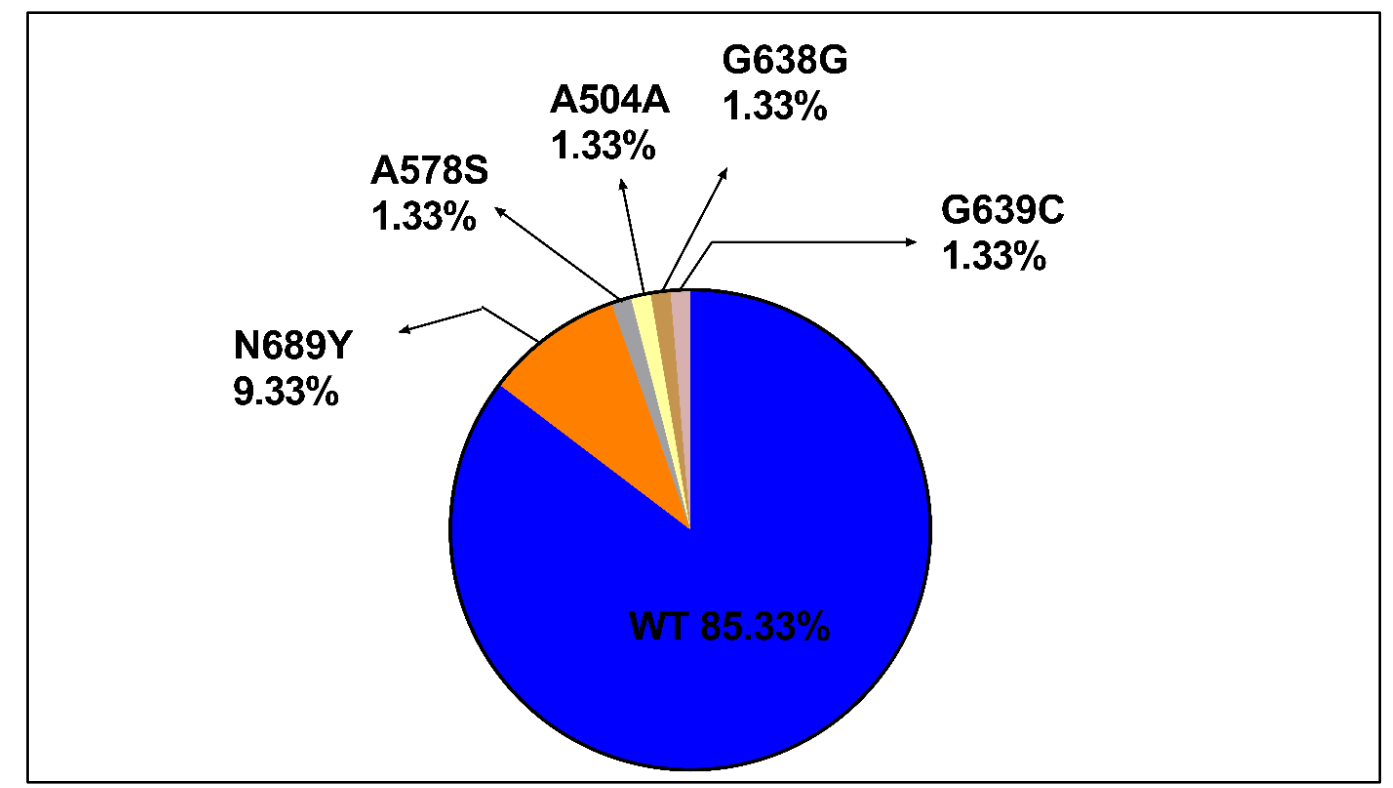

417 
421 Table 3: Evolution of PfKelch13 mutations in $\boldsymbol{P}$. falciparum isolates collected in Dakar 422 2004-2005 vs. 2014-2015.

\begin{tabular}{|l|l|l|l|l|}
\hline \multirow{2}{*}{} & \multicolumn{2}{|c|}{ Pre ACT (n=74) } & \multicolumn{2}{c|}{ Post ACT (n=25) } \\
\hline SNPS & Presence & Frequency (\%) & Presence & Frequency (\%) \\
\hline A578S & + & 1.35 & + & 4 \\
\hline N689Y & + & 9.45 & + & 20 \\
\hline C447Y & - & 0 & + & 8 \\
\hline A504A & + & 1.35 & - & 0 \\
\hline G638G & + & 1.35 & - & 0 \\
\hline G639C & + & 1.35 & - & 0 \\
\hline WT & + & 86 & + & 68 \\
\hline
\end{tabular}



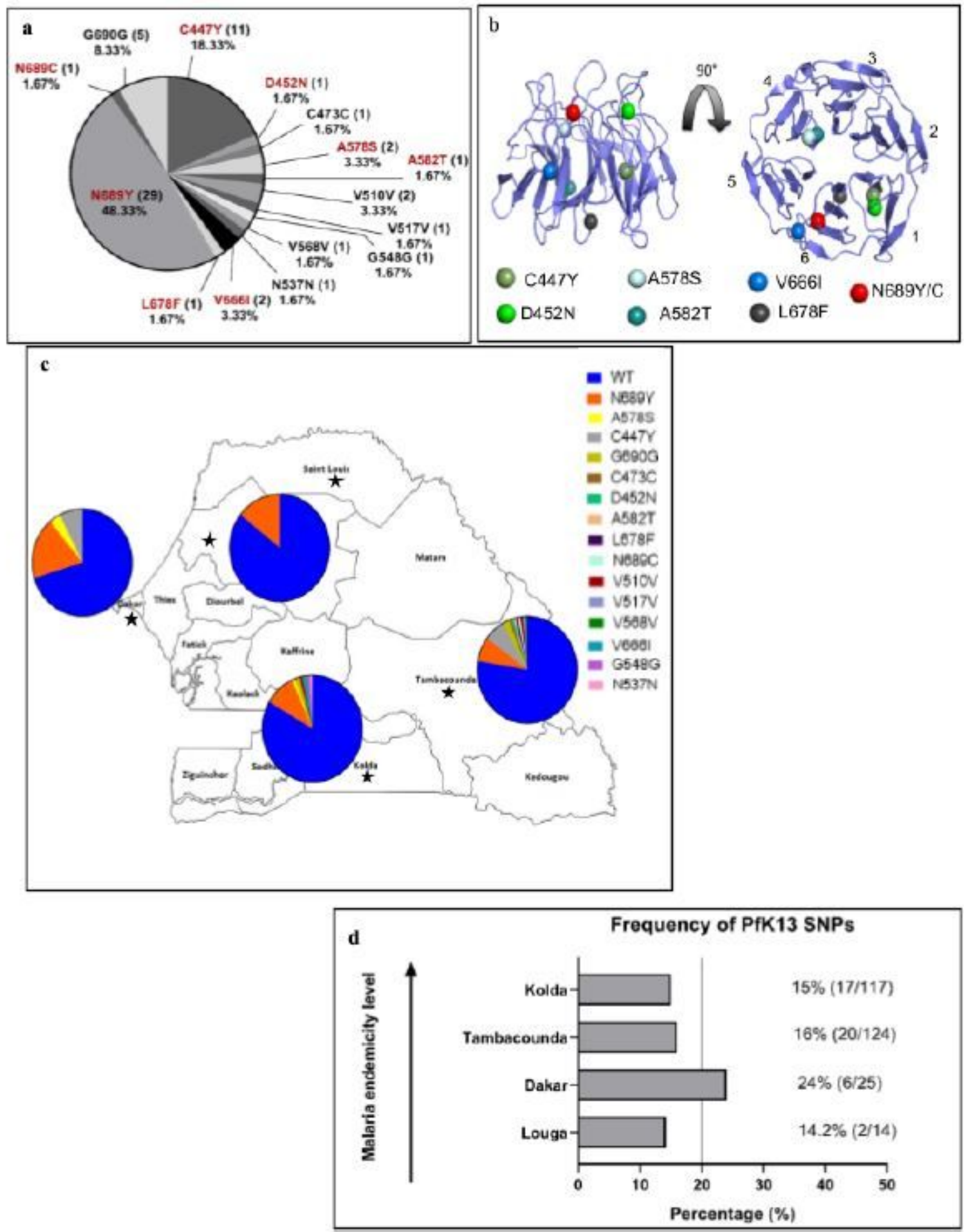

\section{Figure 1}

Geographical distribution of PfKelch13 mutations between 2014 and 2015. a. 15 variants found in 280 clinical isolates are represented. Synonymous mutations are written in black and nonsynonymous mutations are in red. b. Location of the 7 Ns-SNPs on the propeller domain are shown using PfKelch13 
structure pdb code: 4ZGC. c. Mapping of PfKelch13 SNPs in our study sites reveals a high frequency of mutations in highly endemic regions (Kolda and Tambacounda). PfKelch13WT (blue) remains highly frequent in all sites indicating efficacy of ACTs in collected parasite lines. d. Frequency of all SNPs per region is shown. Dakar the capital and low malaria endemic city shows a higher frequency of SNPs with $24 \%$.

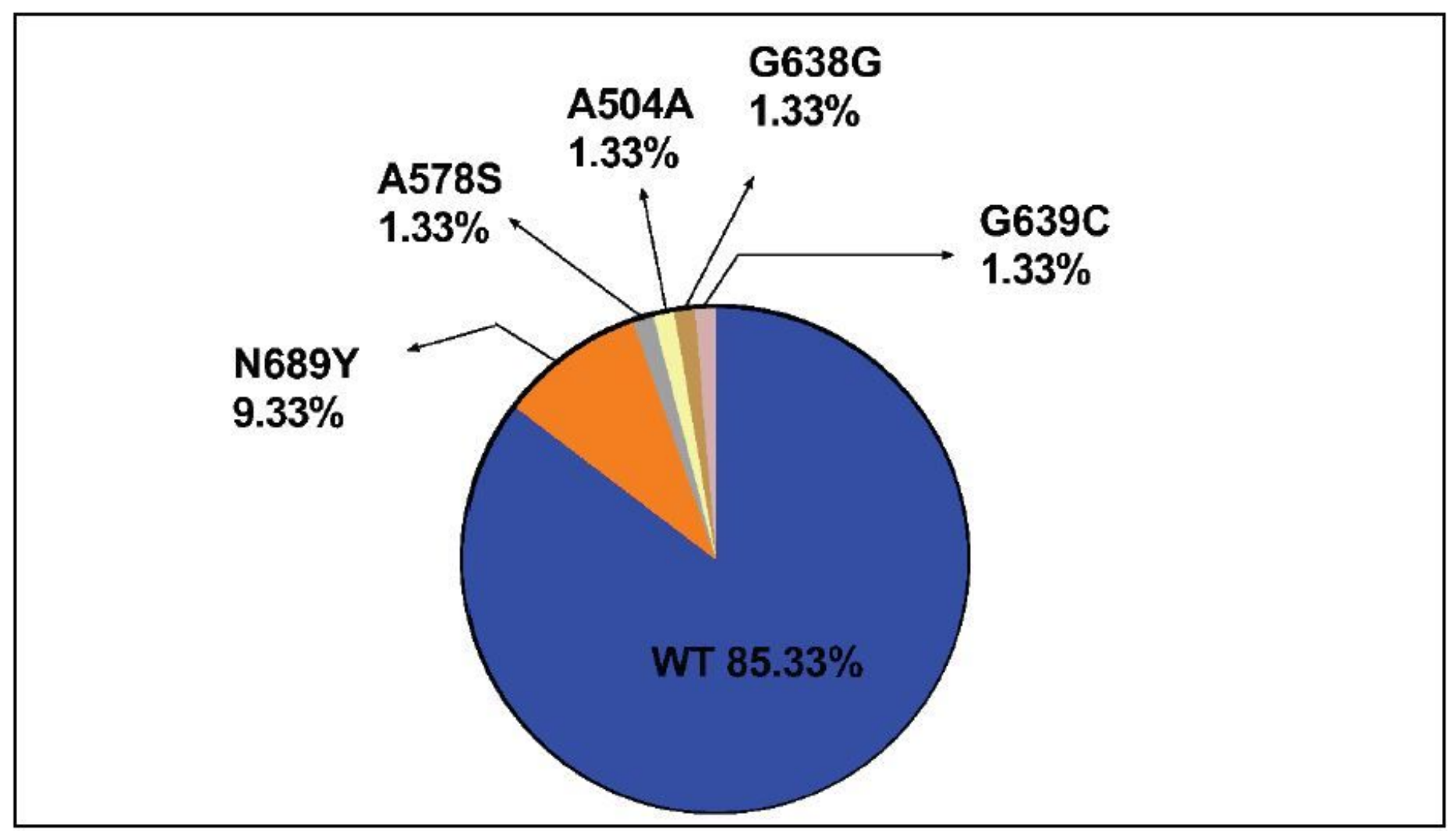

Figure 2

PfKelch13 polymorphism in Dakar in 2004-2005. Prevalence of PfKelch13 priory to ACT introduction reveals in high frequency of PfKelch13WT (85.33\%). Mutations including A578S, N689Y, A504A C638G and $\mathrm{G} 639 \mathrm{C}$ were detectable. None if these variant is associated with artemisinin resistance.

\section{Supplementary Files}

This is a list of supplementary files associated with this preprint. Click to download.

- SupplementaryfileK13ULTIMATE011421.pdf 